\title{
On a functional equation arising from two processors with coupled inputs
}

\author{
El-sayed El-hady ${ }^{1,2 *}$, Wolfgang Förg-Rob ${ }^{1}$ \\ ${ }^{1}$ Fakultät für Mathematik, Informatik und Physik, Universität Innsbruck, A-6020 Innsbruck, Austria \\ ${ }^{2}$ Faculty of Computers and Informatics, Suez Canal University, Ismailis, 41522, Egypt \\ ${ }^{*}$ Corresponding author E-mail: el-sayed.el-hady@uibk.ac.at
}

Copyright (C)2015 El-Sayed El-Hady, Wolfgang Förg-Rob. This is an open access article distributed under the Creative Commons Attribution License, which permits unrestricted use, distribution, and reproduction in any medium, provided the original work is properly cited.

\begin{abstract}
During the last few decades, a certain interesting class of functional equations arises when obtaining the generating functions of many system distributions. Such a class of equations has numerous applications in many modern disciplines like wireless networks and communications. This paper has been motivated by an issue considered by Paul E. Wright in [Advances in applied probability, (1992), 986 - 1007]. The functional equation obtained there has been solved using elliptic functions and analytic continuation, which in turn lead to the determination of the main unknown. Unfortunately that solution seems to be a bit too general with many technical assumptions. In this paper on one hand, we introduce a solution in the symmetric case using boundary value problem approach. On the other hand, we investigate the potential singularities of the unknowns of the functional equation giving one possible application, and we compute some expectation of interest using the corresponding generating function.

Keywords: functional equation, complex analysis, queueing theory, boundary value problem, singularity, generating function.
\end{abstract}

Keywords: 30D05, 97I80, 60K25, 30E25.

\section{Introduction}

Functional equations (FEs) are the equations where the unknowns are functions not variables see $[1,2,3,4,5,6,7]$. Functional equations offer a tool for reducing the mathematical models used to describe many phenomena. The theory of functional equations has thrived principally through the work of the prolific mathematician J. Aczél $[8,9,10,11]$ who identified many of their classes, illustrating efficacious methods for their solutions as well as criteria for the existence and uniqueness of those solutions [6].

FEs arise in models of various fields, such as neural networks [12], economics [13], astronomy [14], population ethics [15], communication see [16], and wireless networks [17]. Specifically, there is no universal solution technique for these FEs, but rather almost each equation is solved differently than the others. The problem of finding some performance measures to some communication, and networks systems comes with a certain interesting class of functional equations.

In general using the literature there are many approaches available to solve such problem of studying the performance evaluation namely, experimental approach, numerical approach, simulation approach, and the analytical approach of course each one has its own advantages and disadvantages see [18] e.g chapter 1 for an overview. This 


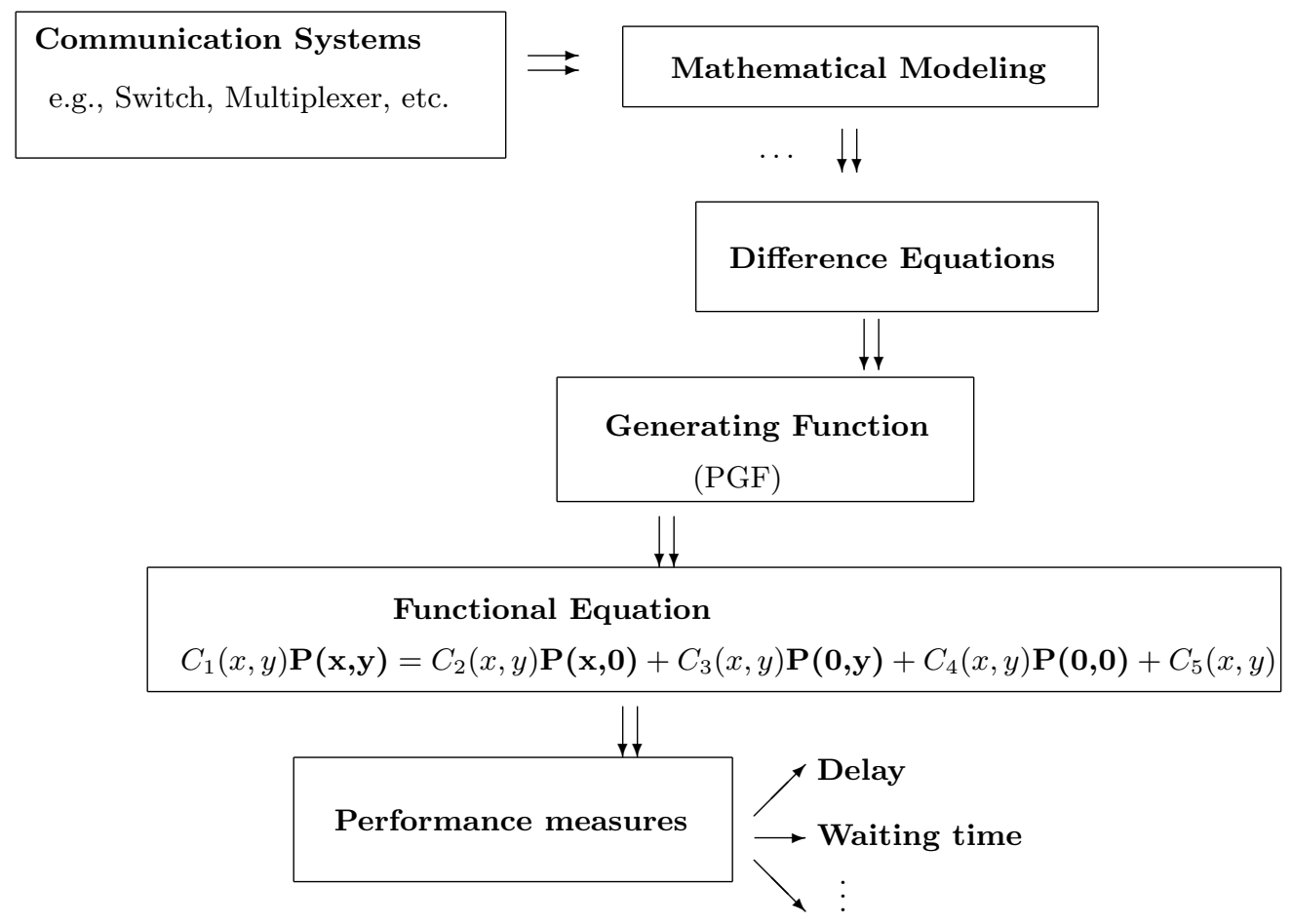

Figure 1: Communication Systems and functional equations-Relation Summary

article is mainly concerned with a solution of a two-variable FE arising from a double queue model originally published in [19] using boundary value problem. Malyshev see [20] was the first person who introduce the approach of transforming functional equations to boundary value problems in the 1970s.

The idea of reducing functional equations for the generating function to a standard Riemann-Hilbert boundary value problem pops up from the work of Fayolle and Iasnogorodski [21] on two parallel $M / M / 1$ queues with coupled processors. A lion share treatments of the boundary value technique for functional equations can be found in Cohen and Boxma [22], Fayolle et al. [23] and [24, 25]. In particular, the general class (1) of two-variable functional equations

$C_{1}(x, y) \mathbf{P}(\mathbf{x}, \mathbf{y})=C_{2}(x, y) \mathbf{P}(\mathbf{x}, \mathbf{0})+C_{3}(x, y) \mathbf{P}(\mathbf{0}, \mathbf{y})+C_{4}(x, y) \mathbf{P}(\mathbf{0}, \mathbf{0})+C_{5}(x, y)$,

where $C_{i}(x, y), i=1,2,3,4,5$ are given polynomials in two complex variables $x, y$, arises from different communication and networks systems.

Special case of (1) arises from wireless networks with stealing see [26]. Similar special case appears in [27] from a tandem queue with coupled processor, another one arises in [17] from rare event asymptotics for a random walk in the quarter plane, and a similar one arises [28] from queueing models with multiple waiting lines. Such equations have been solved by using the theory of boundary value problems [22]. A simple but similar equation appears in [29] has been solved by using Rouchés theorem, and applying the normalization condition. Another special case of such interesting class of equations arises from a lan gateway queueing model see [30]. A similar equation arises from an asymmetric switch see [16].

Figure (1) recapitulates the process between many communication systems on one hand and a certain class of two-variable functional equations on the other hand: Computer scientists who are interested in some communication elements like switches, and they want to find some performance measures like the delay or the waiting time. They start by describing their system mathematically to obtain the mathematical model which in most cases is consisting of a difference equation characterizing the dynamics of the underlying systems together with some boundary conditions. They used a probability generating function (PGF) to map such difference equations to challenged functional equations. Solving such equations will immediately provide the different performance measures of interests.

The sequel of the paper is laid as follows: In the next section we recall the functional equation from the original article [19] together with the solution as originally given in [19] but in brief, in section (3) we solve the equation in the symmetric case by reduction to Riemann-Hilbert boundary value problem, in section (4) we investigate the potential singularity of the unknowns, in section (5) we give one possible application of the singularity, in section 
(6) we compute the expected number of jobs in one queue using the corresponding generating function together with some numerical analysis, and in section (7) we conclude our work.

\section{The Functional Equation}

This equation arises [19] from a double queue arising from a system consisting of two processors serving three job streams, illustrated in figure 2 generated by independent Poisson sources. The central job stream of rate $\nu$ consists of jobs which place resource demands on both processors, which are handled separately by each processor once the request is made. In addition, the first processor receives background work at a rate of $\lambda$ while the second receives similar tasks at a rate $\eta$. Each processor has exponentially distributed service times with rates $\alpha$ and $\beta$ respectively.

The system considered here is a generalization of a two-server system considered by Flatto and Hahn [31] and Flatto [32]. The PGF $P(x, y)$ of the two-dimensional distribution characterizing the system yields the two-variable FE

$Q(x, y) P(x, y)=N(x, y)$,

where

$$
\begin{gathered}
Q(x, y)=\gamma x y-\lambda x^{2} y-\eta x y^{2}-\alpha y-\beta x-\nu x^{2} y^{2}, \\
N(x, y)=\alpha(x-1) y P(0, y)+\beta x(y-1) P(x, 0),
\end{gathered}
$$

and

$$
\gamma \triangleq \alpha+\beta+\nu+\lambda+\eta \text {. }
$$

Equation (2) has been solved by parameterizing the curve $Q(x, y)$ by a pair of elliptic functions $x(\xi)$ and $y(\xi)$ and the relation between $P(x(\xi), 0)$ and $P(0, y(\xi))$ persists on their analytic continuation as elliptic

functions in the $\xi$-plane. The solution is given by

$P(x, y)=\left\{\chi\left(p-\int_{0}^{y} \frac{d \zeta}{\sqrt{\mathscr{E}(\zeta)}}\right)-\chi\left(r-\int_{0}^{x} \frac{d \zeta}{\sqrt{\mathscr{D}(\zeta)}}\right)\right\} \times \frac{(1-x)(1-y)}{Q(x, y)}$

where $\mathscr{E}(), \mathscr{D}()$, are defined in [19], $\chi$ is an elliptic function of order 2, and

$$
r=\int_{0}^{a_{0}} \frac{d \zeta}{\sqrt{\mathscr{D}(\zeta)}}, \quad p=2 \int_{0}^{b_{0}} \frac{d \zeta}{\sqrt{\mathscr{E}(\zeta)}}+r
$$

for some real numbers $a_{0}, b_{0}$. Equation (2) can be rewritten as

$C_{1}(x, y) P(x, y)=C_{2}(x, y) P(x, 0)+C_{3}(x, y) P(0, y)$,

it is obvious that it is a special case of (1) where

$C_{1}(x, y)=\gamma x y-\lambda x^{2} y-\eta x y^{2}-\alpha y-\beta x-\nu x^{2} y^{2}$,

$C_{2}(x, y)=\beta x(y-1)$,

$C_{3}(x, y)=\alpha y(x-1)$,

and

$C_{4}(x, y)=C_{5}(x, y)=0$.

We will investigate the solution of such functional equation based on the service rates $\alpha, \beta$ namely the symmetric case i.e. where $\alpha=\beta$ as follows.

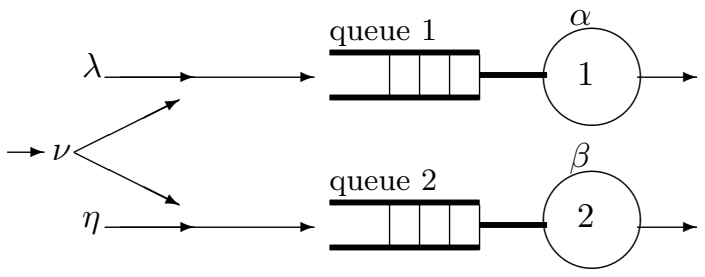

Figure 2: Two Parallel Processors with Coupled Inputs 


\section{The symmetric Case Solution}

Assume that the two processors serve jobs with the same service rate i.e. $\alpha=\beta$. We will use this assumption in equation (3) to introduce a solution of such equation using boundary value problems as follows.

\subsection{The Boundary Value Problem Model of Eq.(3) and its Solution}

In order to solve equation (3), the main idea stems from the fact that the main unknown function $P(x, y)$ is an analytic function in the unit disk, this means that if the $C_{1}(x, y)$ is zero then also the right hand side containing the other unknowns must be zero. Let

$C_{1}(x, y):=\gamma x y-\lambda x^{2} y-\eta x y^{2}-\alpha y-\alpha x-\nu x^{2} y^{2}=0$,

then also

$\alpha x(y-1) P(x, 0)+\alpha y(x-1) P(0, y)=0$.

The solution of the original functional equation (3) is now reduced to the solution of the functional equation (6). Dividing equation $(6)$ by $(x-1)(y-1) \neq 0$ to get

$\frac{\alpha x}{x-1} P(x, 0)+\frac{\alpha y}{y-1} P(0, y)=0$.

Introduce the function

$$
g(x):=\frac{\alpha x}{x-1} P(x, 0)
$$

then equation (7) can be rewritten as

$g(x)+g(y)=0$,

where the function $g($.$) is an analytic function except for a simple pole at 1$.

Now we reduced the solution of the main functional equation to the solution of $(8)$ in $\left\{(x, y): C_{1}(x, y)=0\right\}$. But $C_{1}(x, y)=0$ offers a very large number of ordered pairs, in our symmetric case it is natural to consider the set:

$$
M^{*}:=\left\{(x, \tilde{x}): C_{1}(x, \tilde{x})=0\right\},
$$

where $\tilde{x}$ stands for the complex conjugate of $x$. Using this special set we can rewrite equation (8) in the form

$g(x)+g(\tilde{x})=0 \rightarrow \Re g(x)=0$,

where $\Re$ sands or the real part of the complex variable. Now we have a boundary value problem:

The problem of determining a function $g($.$) which satisfies:$

1. Analytic everywhere except for a simple pole at 1

2. $\Re g()=$.0 on $M \backslash\{1\}$

3. $\lim _{x \rightarrow 1}(x-1) g(x)=\alpha P(1,0)=\alpha-\nu-\eta$

where $P(1,0)=\frac{\alpha-\nu-\eta}{\alpha}$ is obtained from the main equation by using the normalization condition $P(1,1)=1$. In order to solve the boundary value problem constructed, let $\phi$, with inverse $\psi$, be the conformal mapping see e.g. [33, 34] of the unit disk onto the region bounded by $M^{*}$ with normalization conditions $\phi(0)=K$, $\phi(1)=1$ for some $K$.

This mapping exists by the Riemann mapping theorem see [35], the curve $M^{*}$ is simply connected for some special values of the parameters $\alpha, \gamma, \nu, \eta, \lambda$. Define $h(w):=g(\phi(w))$. We then obtain a relatively simple Riemann Hilbert boundary value problem with a pole, for $h($.$) on the unit circle D$ (actually, it is a Dirichlet problem with a pole see [22]):

1. $\Re h(w)=0$, on $w \in D \backslash\{1\}$

2. $\lim _{w \rightarrow 1}(w-1) h(w)=\frac{\alpha-\nu-\eta}{\phi(1)}$, where $\dot{\phi}()=.\frac{d \phi}{d x}$ 
3. $h($.$) analytic on D$, continuous on $\bar{D} \backslash\{1\}$.

The solution of this boundary value problem is

$h(w)=\frac{1}{2} \frac{\alpha-\nu-\eta}{\dot{\phi}(1)} \frac{w+1}{w-1}, \quad w \in D$

which determines

$g(x)=h(\psi(x))=\frac{1}{2} \frac{\alpha-\nu-\eta}{\dot{\phi}(1)} \frac{\psi(x)+1}{\psi(x)-1}$,

inside the curve $M$; Substitution in the original equation finally implies

$P(x, y)=(\alpha-\nu-\eta) \psi^{\prime}(1) \frac{(x-1)(y-1)}{(\psi(x)-1)(\psi(y)-1)} \times \frac{\psi(x) \psi(y)-1}{\gamma x y-\lambda x^{2} y-\eta x y^{2}-\alpha y-\alpha x-\nu x^{2} y^{2}}$

\section{Singularities of the Unknowns}

The main idea of locating the possible singularities of the unknowns of the functional equation (3) will be solely to find the intersection points between two functions. So that it is legitimate first to introduce the idea of computing the intersection points between two general functions. Generally speaking, when we have two polynomials in two variables,

$f_{1}(x, y)=a_{0}(y)+a_{1}(y) x+\cdots+a_{m}(y) x^{m}$,

and

$f_{2}(x, y)=b_{0}(y)+b_{1}(y) x+\cdots+b_{n}(y) x^{n}$,

the resultant $\operatorname{Res}_{x}\left(f_{1}, f_{2}\right)$ of the polynomials $f_{1}$ and $f_{2}$ with respect to $x$ is the determinant given by (see e.g. [36] appendix C, and [37]).

$\left.\left|\begin{array}{ccccccc}a_{m} & a_{m-1} & \cdots & a_{0} & 0 & \cdots & \cdots \\ 0 & a_{m} & a_{m-1} & \cdots & a_{0} & 0 & \cdots \\ \cdots & \cdots & \cdots & \cdots & \cdots & \cdots & \cdots \\ \cdots & \cdots & 0 & a_{m} & a_{m-1} & \cdots & a_{0} \\ b_{n} & b_{n-1} & \cdots & b_{0} & 0 & \cdots & \cdots \\ 0 & b_{n} & b_{n-1} & \cdots & b_{0} & 0 & \cdots \\ \cdots & \cdots & \cdots & \cdots & \cdots & \cdots & \cdots \\ \cdots & \cdots & 0 & b_{n} & b_{n-1} & \cdots & b_{0}\end{array}\right|\right\} m$ rows

which is a polynomial in $y$. The polynomials $f_{1}$ and $f_{2}$ have a common nontrivial root $\left(x_{0}, y_{0}\right)$ if and only if the resultant with respect to $x$ is 0 at $y_{0}$.

\section{1. $\quad$ Singularity of $P(x, 0)$}

Since the main PGF $(\mathrm{P}(\mathrm{x}, \mathrm{y}))$ in (3) is by definition an analytic function in the unit disk, this means that if $C_{1}(x, y)=0$, then also

$C_{2}(x, y) P(x, 0)+C_{3}(x, y) P(0, y)=0$,

which is equivalent to

$P(x, 0)=-\frac{C_{3}(x, y)}{C_{2}(x, y)} P(0, y)$.

We observe from the above equation that the unknown function $f(x)$ could have a singularity at some point $y$ if for some $x, C_{1}(x, y)=0$ and $C_{2}(x, y)=0$. This leads us to study the intersection points of the curves $C_{1}(x, y)=0$ and $C_{2}(x, y)=0$. If such a point $x$ exists, then $y$ is a root of the resultant $\operatorname{Res}_{x}\left(h_{1}, h_{2} ; y\right)$ in $x$ of the polynomials $C_{1}(x, y)$ and $C_{2}(x, y)$. 


\subsection{1. $\quad$ Resultant in $x$}

The two functions $C_{1}(x, y)$ and $C_{2}(x, y)$ can be rewritten as functions in $x$ as follows:

$$
\begin{aligned}
C_{1}(x, y) & =\nu x^{2} y^{2}+\lambda x^{2} y+\eta x y^{2}+\alpha y+\beta x-\gamma x y \\
& =\alpha y+\left(\eta y^{2}+\beta-\gamma y\right) x+\left(\lambda y+\nu y^{2}\right) x^{2} \\
& =a_{0}(y)+a_{1}(y) x+a_{2}(y) x^{2},
\end{aligned}
$$

where

$a_{2}(y)=\lambda y+\nu y^{2}$,

$a_{1}(y)=\eta y^{2}+\beta-\gamma y$,

$a_{0}(y)=\alpha y$,

and

$$
\begin{aligned}
C_{2}(x, y) & =\beta x(y-1) \\
& =(\beta y-\beta) x \\
& =b_{0}(y)+b_{1}(y) x,
\end{aligned}
$$

where

$b_{1}(y)=\beta y-\beta$,

and

$b_{0}(y)=0$.

Using (11), (12), and (13), the resultant in $x$ is the determinant of the matrix,

$A=\left(\begin{array}{ccc}a_{2} & a_{1} & a_{0} \\ b_{1} & b_{0} & 0 \\ 0 & b_{1} & b_{0}\end{array}\right)$

From (14) after some algebraic manipulations we can write that

$$
\begin{aligned}
\operatorname{Res}_{x}\left(C_{1}, C_{2} ; y\right) & =|A|=a_{2}\left|\begin{array}{cc}
b_{0} & 0 \\
b_{1} & b_{0}
\end{array}\right|-b_{1}\left|\begin{array}{cc}
a_{1} & a_{0} \\
b_{1} & b_{0}
\end{array}\right| \\
& =a_{2} b_{0}^{2}-b_{1}\left(a_{1} b_{0}-a_{0} b_{1}\right) \\
& =0-(\beta y-\beta)(0-\alpha y(\beta y-\beta)) \\
& =\alpha \beta^{2} y(y-1)^{2}
\end{aligned}
$$

so that we conclude that the function $f(x)$ has a possible singularity in $x$ as a function of $y$ at $y_{a}:=0,1,1,$. Since $y_{a}$ is a zero of $C_{1}(x, y)$, therefore solving $C_{1}\left(x, y_{a}\right)=0$ to get $x_{a}$.

\subsubsection{Resultant in $y$}

The two functions $C_{1}(x, y)$ and $C_{2}(x, y)$ can be rewritten as functions in $y$ as follows:

$$
\begin{aligned}
C_{1}(x, y) & =\nu x^{2} y^{2}+\lambda x^{2} y+\eta x y^{2}+\alpha y+\beta x-\gamma x y \\
& =\beta x+\left(\lambda x^{2}-\gamma x+\alpha\right) y+\left(\nu x^{2}+\eta x\right) y^{2} \\
& =c_{0}(x)+c_{1}(x) y+c_{2}(x) y^{2},
\end{aligned}
$$

where

$c_{2}(x)=\nu x^{2}+\eta x$, 
$c_{1}(x)=\lambda x^{2}-\gamma x+\alpha$,

$c_{0}(x)=\beta x$,

and

$$
\begin{aligned}
C_{2}(x, y) & =\beta x(y-1) \\
& =-\beta x+\beta x y \\
& =d_{0}(x)+d_{1}(x) y+d_{2}(x) y^{2},
\end{aligned}
$$

where

$d_{2}(x)=0$,

$d_{1}(x)=\beta x$,

and

$d_{0}(x)=-\beta x$.

Using (11), (15), and (16) the resultant in $y$ is the determinant of the matrix,

$B=\left(\begin{array}{ccc}c_{2} & c_{1} & c_{0} \\ d_{2} & d_{1} & d_{0} \\ 0 & d_{2} & d_{1}\end{array}\right)$

From (17) after some algebraic manipulations we can write that

$$
\begin{aligned}
\operatorname{Res}_{y}\left(C_{1}, C_{2} ; x\right) & =|B|=c_{2}\left|\begin{array}{cc}
d_{1} & d_{0} \\
d_{2} & d_{1}
\end{array}\right|-d_{2}\left|\begin{array}{cc}
c_{1} & c_{0} \\
d_{2} & d_{1}
\end{array}\right| \\
& =c_{2}\left(d_{1}^{2}-d_{0} d_{2}\right)-d_{2}\left(c_{1} d_{1}-c_{0} d_{2}\right) \\
& =\left(\nu x^{2}+\eta x\right)(\beta x)^{2} \\
& =\beta^{2}(\nu x+\eta) x^{3}
\end{aligned}
$$

we can conclude that the function $P(x, 0)$ has a possible singularity in $y$ as a function of $x$ at $x=0$, and at $x=-\frac{\eta}{\nu}$. Combining the results of the previous subsection i.e. $x_{a}$ with the current one, so that we conclude that the function $f(x)$ has a possible singularity in $x$ at:

1. $x=0$ of multiplicity three

2. $x=x_{a}$

3. $x=-\frac{\eta}{\nu}$

In a complete symmetric manner to sect. 4.1 we can compute the potential singularity of the function $P(0, y)$.

\section{Application of Singularity}

One possible application of computing the singularities of the unknowns of functional equations is to obtain the asymptotic behavior of the sequences defined by such unknowns. In general, asymptotic analysis endeavors are to find a solution that closely approximates the exact solution see e.g. [38]. The functional equation of our interest is in fact an equation in which the unknowns are generating functions namely, $P(x, y), P(x, 0), P(0, y)$ with interesting coefficients $p_{m, n}, p_{m, 0}, p_{0, n}$ respectively, that have no simple closed form. In this section we give pointers to a technique for obtaining the asymptotic of the marginal distribution $p_{m, 0}$ for large $m$, given $P(x, 0)=\sum_{m=0}^{\infty} p_{m, 0} x^{m}$. If the function $P(x, 0)$ has singularities, then Darboux's theorem can use these singularities to estimate the sequence $p_{m, 0}$. The statement of the theorem is see [18] chapter 1 as follows. 
Theorem 1 (Darboux's method). Suppose $P(x, 0)=\sum_{m=0}^{\infty} p_{m, 0} x^{m}$ with positive real coefficients $p_{m, 0}$ is analytic near 0 and has only algebraic singularities $x_{k}$ on its circle of convergence $|x|=R$, in other words, in a neighborhood of $x_{k}$ we have

$$
P(x, 0) \approx\left(1-\frac{x}{x_{k}}\right)^{-w_{k}} G_{k}(x),
$$

where $w_{k} \neq 0,-1,-2, \cdots$ and $G_{k}(x)$ denotes a nonzero analytic function near $x_{k}$. Let $w=\max _{k} \Re\left(w_{k}\right)$ denote the maximum of the real parts of the $w_{k}$. Then we have

$$
p_{m, 0}=\sum_{j} \frac{G_{j}\left(x_{j}\right)}{\Gamma\left(w_{j}\right)} m^{w_{j}-1} x_{j}^{-n}+o\left(m^{w-1} R^{-m}\right)
$$

with the sum taken over all $j$ with $\Re\left(w_{j}\right)=w$ and $\Gamma(w)$ the Gamma function of $w($ with $\Gamma(n)=(n-1)$ ! for $n$ a positive integer).

\section{Expectations and Numerical Analysis}

In this section we find the expected number of jobs in one of the queues using the corresponding generating functions. It is easy to see using (3) that the generating function of the number of jobs in the first queue is given by

$$
\begin{aligned}
P(x, 1)= & \sum_{m=0}^{\infty} P\left(N_{1}=m\right) x^{m} \\
& =\frac{\alpha(x-1) P(0,1)}{\left(\gamma x-\lambda x^{2}-\eta x-\alpha-\beta x-\nu x^{2}\right)},
\end{aligned}
$$

where $N_{1}$ is the number of files in the first queue. Using the normalization condition $P(1,1)=1$ in the above

\begin{tabular}{|l|l|l|}
\hline$\alpha$ & $\nu$ & $E\left[N_{1}\right]$ \\
\hline 2 & 0,3 & 0,5384 \\
4 & 0,5 & 0,25 \\
6 & 0,6 & 0,1764 \\
8 & 0,7 & 0,14285 \\
10 & 0,8 & 0,12359 \\
12 & 0,88 & 0,10905 \\
14 & 0,9 & 0,09375 \\
16 & 0,92 & 0,08254 \\
18 & 0,93 & 0,07334 \\
20 & 0,94 & 0,06609 \\
22 & 0,97 & 0,06126 \\
30 & 0,978 & 0,04449 \\
40 & 0,98 & 0,03305 \\
70 & 0,99 & 0,01877 \\
\hline
\end{tabular}

TABLE I: The expected number of jobs, the service rate $\alpha$, the arrival rate $\nu$ when $\lambda=0.4$

equation to find $P(0,1)$ after some nontrivial manipulations including applying de l'Hospitals rule to get

$P(0,1)=\frac{\alpha-\nu-\lambda}{\alpha}$.

From (18) we will compute the expected number of jobs in queue 1. It is well known see e.g. [29] that the expected number of jobs in queue 1 is given by

$$
\begin{aligned}
E\left[N_{1}\right] & =\left.\frac{\partial}{\partial x} P(x, 1)\right|_{x=1} \\
& =\left.\frac{\partial}{\partial x} \frac{P(0,1) \alpha(x-1)}{\left(\gamma x-\lambda x^{2}-\eta x-\alpha-\beta x-\nu x^{2}\right)}\right|_{x=1} \\
& =\frac{0}{0},
\end{aligned}
$$


therefore by applying de L'Hôpital's rule two times to end up with

$E\left[N_{1}\right]=P(0,1) \frac{\alpha[\lambda+\nu]}{(\gamma-2 \lambda-\eta-\beta-2 \nu)^{2}}$.

Using (19) the expected number of jobs in the first queue is given by

$E\left[N_{1}\right]=\frac{(\alpha-\nu-\lambda)(\lambda+\nu)}{(\gamma-2 \lambda-\eta-\beta-2 \nu)^{2}}$,

or after using the relation between $\gamma$ and the other parameters to get that

$E\left[N_{1}\right]=\frac{\lambda+\nu}{\alpha-\lambda-\nu}$.

which is the expected number of jobs in the first queue of the queueing system.

\begin{tabular}{|l|l|l|}
\hline$\alpha$ & $\nu$ & $E\left[N_{1}\right]$ \\
\hline 2 & 0,3 & 1,2222 \\
4 & 0,5 & 0,2500 \\
6 & 0,6 & 0,1765 \\
8 & 0,7 & 0,1429 \\
10 & 0,8 & 0,1236 \\
12 & 0,88 & 0,1091 \\
14 & 0,9 & 0,0938 \\
16 & 0,92 & 0,0825 \\
18 & 0,93 & 0,0733 \\
20 & 0,94 & 0,0661 \\
22 & 0,97 & 0,0613 \\
30 & 0,978 & 0,0445 \\
40 & 0,98 & 0,0331 \\
50 & 0,99 & 0,0265 \\
\hline
\end{tabular}

TABLE II: The expected number of jobs, the service rate $\alpha$, and the arrival rate $\nu$ when $\lambda=0.8$

\begin{tabular}{|l|l|l|}
\hline$\lambda$ & $\nu$ & $E\left[N_{1}\right]$ \\
\hline 0,01 & 0,3 & 0,0265 \\
0,2 & 0,35 & 0,0480 \\
0,3 & 0,42 & 0,0638 \\
0,4 & 0,43 & 0,0743 \\
0,5 & 0,53 & 0,0939 \\
0,6 & 0,62 & 0,1132 \\
0,7 & 0,68 & 0,1299 \\
0,8 & 0,7 & 0,1429 \\
0,9 & 0,76 & 0,1605 \\
0,92 & 0,88 & 0,1765 \\
0,96 & 0,92 & 0,1858 \\
0,98 & 0,95 & 0,1917 \\
\hline
\end{tabular}

TABLE III: The expected number of jobs, the arrival rate $\lambda$, and the arrival rate $\nu$ when $\alpha=12$

The numerical results generated in table I are obtained from equation (20) for fixed arrival rate $\lambda=0.4$. In table I we generate the results for fixed arrival rate $\lambda=0.8$. In tables III, IV we fix the service rate $\alpha=12,5$ respectively. In figure 3, 4, 5, 6 we plot the results of table I, II, III, IV respectively. It is clear that in figures 3 , 4 for fixed arrival rate $\lambda=0.4,0.8$ the higher the service rate the lower the expected number of jobs. This makes perfect sense because when we have a higher service rate the number of jobs waiting will dramatically decrease. In figures 5,6 when fixing the service rates $\alpha=12,5$ the higher the arrival rates $\lambda, \nu$ the higher the expected number 


\begin{tabular}{|l|l|l|}
\hline$\lambda$ & $\nu$ & $E\left[N_{1}\right]$ \\
\hline 0,01 & 0,3 & 0,06609 \\
0,2 & 0,4 & 0,136363636 \\
0,3 & 0,42 & 0,168224 \\
0,4 & 0,43 & 0,19904 \\
0,5 & 0,53 & 0,25944 \\
0,6 & 0,62 & 0,32275 \\
0,7 & 0,68 & 0,381215 \\
0,8 & 0,7 & 0,42857 \\
0,9 & 0,76 & 0,497005 \\
0,92 & 0,88 & 0,5625 \\
0,96 & 0,92 & 0,60256 \\
0,99 & 0,99 & 0,6556 \\
\hline
\end{tabular}

TABLE IV: The expected number of jobs, the arrival rate $\lambda$, and the arrival rate $\nu$ when $\alpha=5$

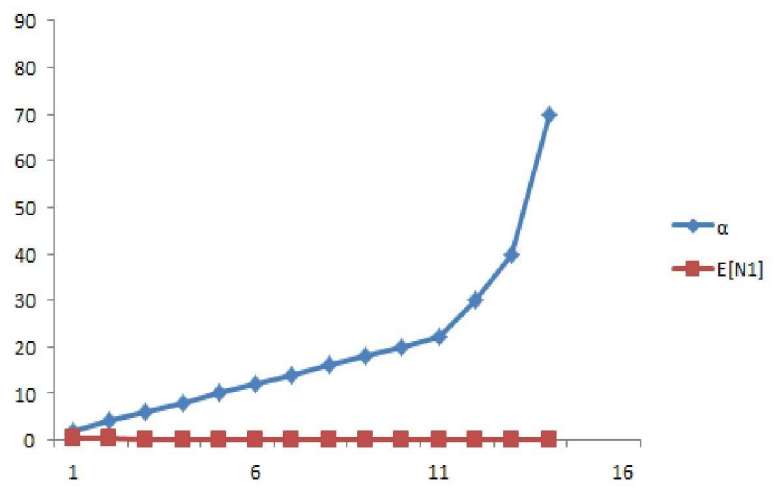

Figure 3: The expected number of jobs vs. the service rate for fixed arrival rate $\lambda=0.4$

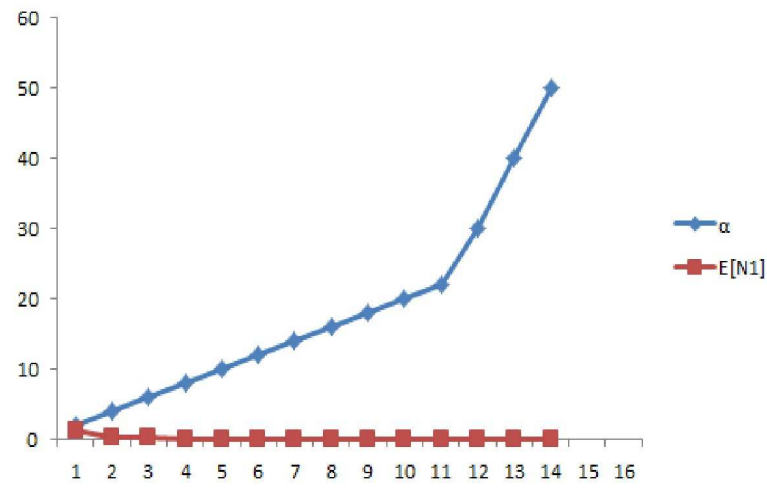

Figure 4: The expected number of jobs vs. the service rate for fixed arrival rate $\lambda=0.8$ 


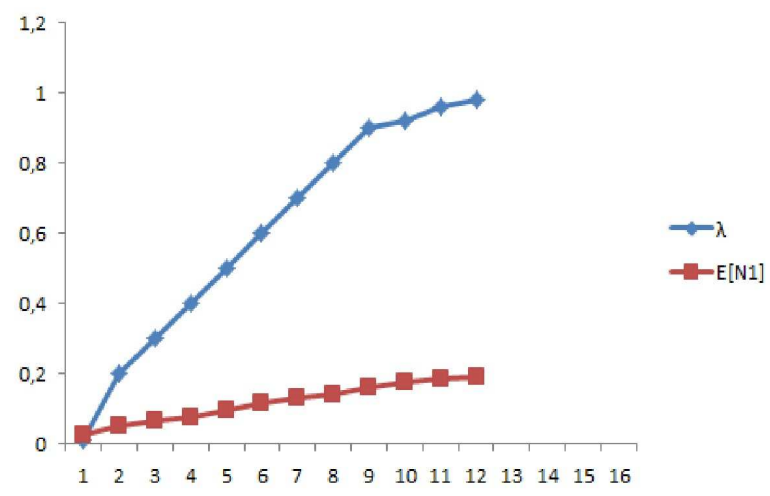

Figure 5: The expected number of jobs vs. the arrival rate for fixed service rate $\alpha=12$

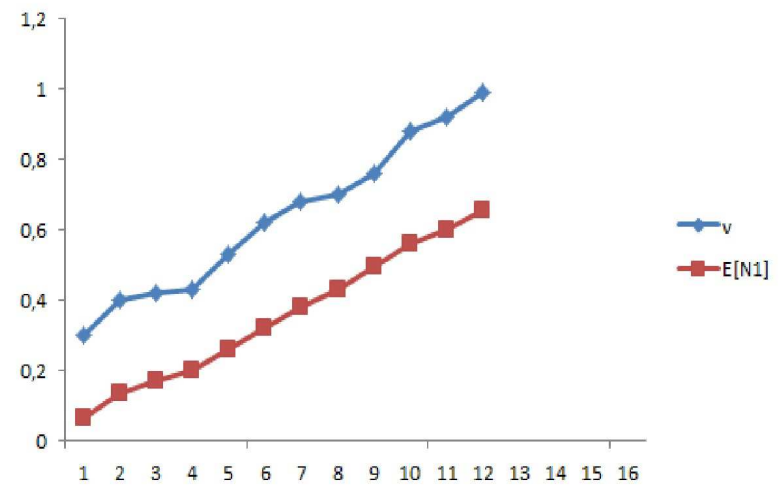

Figure 6: The expected number of jobs vs. the arrival rate for fixed service rate $\alpha=5$

of jobs which makes also perfect sense. In a complete symmetric way we can obtain the expected number of jobs in the second queue using the corresponding generating function, and of course we can generate the corresponding numerical results. 


\section{Conclusion}

In this paper, we managed to introduce a solution of a two-variable functional equation arising from a queueing model of a system consisting of two parallel processors with coupled inputs. This is done by considering the symmetric case on the underlying system and by reduction to a boundary value problem. One more contribution is the investigation of the possible singularities of the unknowns and the computation of some expectation. Possible extension of this work could be to solve the equation in the asymmetric case using boundary value problems approach, and to introduce a general solution methodology to such interesting class of equations.

\section{Funds}

The first author is a joint-scholarship holder. Such a scholarship is fully funded by the Egyptian ministry of higher education and scientific research, Cairo, EGYPT during the period January 25, 2013 till January 24, 2015. The author also gets a young-talent scholarship number 2014/3/MIP - 12 funded by Innsbruck University, Austria during the period March 1, 2015 till August 31, 2015.

\section{References}

[1] P. K. Sahoo and P. Kannappan, Introduction to functional equations, CRC Press, 2011.

[2] M. Kuczma, "A survey of the theory of functional equations", Univ. Beograd Publ. Elektrotechn. Fak. Ser.Mat.Fiz, No.130, (1964), pp. 1-64.

[3] M. Kuczma, An introduction to the theory of functional equations and inequalities: Cauchy's equation and Jensen's inequality, Springer, 2008.

[4] C. G. Small, Functional equations and how to solve them, Springer, 2007.

[5] T. M. Rassias and J. Brzdek, Functional Equations in Mathematical Analysis, Springer, 2012.

[6] J. Aczél, Lectures on functional equations and their applications, Courier Dover Publications, 2006.

[7] Z. Daróczy and Z. Páles, Functional Equations-Results and Advances, Springer, 2002.

[8] J. Aczél and J. G. Dhombres, Functional equations in several variables, Birkhauser, Cambridge University Press, USA, 31, 1989.

[9] J. Aczél, Short Course on Functional Equations: Based Upon Recent Applications to the Social and Behavioural Sciences, Springer Science \& Business Media, Vol. 3, (1987).

[10] J. Aczel, J. C. Falmagne and R. D. Luce, "Functional equations in the behavioral sciences^1", Mathematica japonicae, Vol. 52, No. 3, pp. 469-512, (2000).

[11] J. Aczél, On applications and theory of functional equations, Academic Press, 2014.

[12] L. Kindermann, A. Lewandowski, and P. Protzel, "A framework for solving functional equations with neural networks", Proceedings of Neural Information Processing (ICONIP2001), 2, (2001), pp. 1075-1078.

[13] W. Gehrig, Functional equation methods applied to economic problems: some examples, Functional Equations: History, Applications and Theory, Springer, pp. 33-52, (1984).

[14] H. Fripertinger and J. Schwaiger, Some applications of functional equations in astronomy, na, (2001)

[15] C. Blackorby, W. Bossert and D. Donaldson, "Functional equations and population ethics", aequationes mathematicae, Vol. 58, No. 3, (1999), pp. 272-284.

[16] J. Cohen, "On the asymmetric clocked buffered switch", Queueing systems, Springer, Vol. 30, No. 3-4, (1998), pp. $385-404$.

[17] F. Guillemin and J. S. V. Leeuwaarden, "Rare event asymptotics for a random walk in the quarter plane", Queueing Systems, Springer, Vol. 67, No. 1, (2011), pp. 65-98. 
[18] J. Walraevens, Discrete-time queueing models with priorities, Ghent University, (2004).

[19] P. E. Wright, "Two parallel processors with coupled inputs", Advances in applied probability, JSTOR, (1992), pp. 986-1007.

[20] V. A. Malyshev, "An analytical method in the theory of two-dimensional positive random walks", Siberian Mathematical Journal, Springer, Vol. 13, No. 6, (1972), pp. 917-929.

[21] G. Fayolle and R. Iasnogorodski, "Two coupled processors: the reduction to a Riemann-Hilbert problem", Zeitschrift für Wahrscheinlichkeitstheorie und verwandte Gebiete, Springer, Vol. 47, No. 3, (1979), pp. 325-351.

[22] J. W. Cohen and O. J. Boxma, Boundary value problems in queueing system analysis, Elsevier, 2000.

[23] G. Fayolle, R. Iasnogorodski, and V. A. Malyshev, Random walks in the quarter-plane: algebraic methods, boundary value problems and applications, Vol. 40, Springer, (1999).

[24] N. I. Muskhelishvili, Singular integral equations: boundary problems of function theory and their application to mathematical physics, Courier Dover Publications, 2008.

[25] F. D. Gakhov, Boundary value problems, Courier Corporation, 1990.

[26] F. Guillemin, C. Knessl, and J. S. V. Leeuwaarden, "Wireless Multihop Networks with Stealing: Large Buffer Asymptotics via the Ray Method", SIAM Journal on Applied Mathematics, SIAM, Vol. 71, No. 4, (2011), pp. 1220-1240.

[27] J. Resing and L. ÖRmeci, "A tandem queueing model with coupled processors", Operations Research Letters, Vol. 31, No. 5, Elsevier, (2003), pp. 383-389.

[28] I. J. Adan, O. J. Boxma, and J. Resing, "Queueing models with multiple waiting lines", Queueing Systems, Springer, Vol. 37, No. 1-3, (2001), pp. 65-98.

[29] H. Nassar, H. Al Mahdi, "Queueing analysis of an ATM multimedia multiplexer with non-pre-emptive priority", IEE Proceedings-Communications, IET, Vol. 150, No. 3, (2003), pp. 189-196.

[30] H. Nassar, "Two-dimensional queueing model for a LAN gateway", WSEAS Transactions on Communications, WSEAS PRESS, Vol. 5, No. 9, (2006), pp. 1585.

[31] L. Flatto and S. Hahn, "Two parallel queues created by arrivals with two demands I", SIAM Journal on Applied Mathematics, Vol. 44, No. 5, (1984), pp. 1041-1053.

[32] L. Flatto, "Two parallel queues created by arrivals with two demands II", SIAM Journal on Applied Mathematics, Vol. 45, No. 5, pp.861-878, (1985).

[33] Z. Nehari, Conformal mapping, Courier Corporation, 1975.

[34] H. Kober, Dictionary of conformal representations, Dover New York, Vol. 2, 1957.

[35] R. P. Agarwal, K. Perera and S. Pinelas, An introduction to complex analysis, Springer, 2011.

[36] P. Flajolet and R. Sedgewick, Analytic combinatorics, Cambridge University press, 2009.

[37] E-s. El-Hady, W. Förg-Rob, H. Nassar, and M. A. W. Mahmoud, On a functional equation arising from a difference equation characterizing the dynamics of a network gateway, Submitted for publication, (2015).

[38] D. H. Greene and D. E. Knuth, Mathematics for the Analysis of Algorithms, Springer Science EJ Business Media, 2007. 